\title{
Catch and Release of Microwave Photon States
}

\author{
Yi Yin, ${ }^{1, *}$ Yu Chen, ${ }^{1}$ Daniel Sank, ${ }^{1}$ P. J. J. O’Malley, ${ }^{1}$ T. C. White,${ }^{1}$ R. Barends, ${ }^{1}$ J. Kelly, ${ }^{1}$ Erik Lucero, ${ }^{1}$ \\ Matteo Mariantoni, ${ }^{1,2}$ A. Megrant,${ }^{3}$ C. Neill, ${ }^{1}$ A. Vainsencher, ${ }^{1}$ J. Wenner, ${ }^{1}$ Alexander N. Korotkov, ${ }^{4}$ A. N. Cleland, ${ }^{1,2}$ and \\ John M. Martinis ${ }^{1,2, \dagger}$ \\ ${ }^{1}$ Department of Physics, University of California, Santa Barbara, California 93106, USA \\ ${ }^{2}$ California NanoSystems Institute, University of California, Santa Barbara, California 93106, USA \\ ${ }^{3}$ Department of Materials, University of California, Santa Barbara, California 93106, USA \\ ${ }^{4}$ Department of Electrical Engineering, University of California, Riverside, California 92521, USA
}

(Received 18 October 2012; published 4 March 2013)

\begin{abstract}
We demonstrate a superconducting resonator with variable coupling to a measurement transmission line. The resonator coupling can be adjusted through zero to a photon emission rate 1000 times the intrinsic resonator decay rate. We demonstrate the catch and release of photons in the resonator, as well as control of nonclassical Fock states. We also demonstrate the dynamical control of the release waveform of photons from the resonator, a key functionality that will enable high-fidelity quantum state transfer between distant resonators or qubits.
\end{abstract}

DOI: 10.1103/PhysRevLett.110.107001

PACS numbers: 85.25.Cp, 03.67.Lx, 42.50.Gy

Superconducting resonators play a central role in quantum information technology. Applications include the synthetic generation and storage of photon quantum states [1-3], quantum memories for quantum computation [4], and dispersive measurements of superconducting qubits $[5,6]$, as well as defects in diamond [7,8]. Resonators with low internal losses are typically desirable, but the resonator's coupling strength to the quantum system and to its measurement apparatus is application dependent. When coupling a resonator to a qubit, either for a quantum memory or in a circuit quantum electrodynamics [5] experiment, strong coupling to the qubit improves information transfer but also increases dephasing. When reading out a qubit, coupling the resonator strongly to its measurement apparatus increases the measurement bandwidth and signal but in addition increases dissipation [9]. Resonator designs therefore involve compromises between the competing needs for both strong and weak coupling [10,11]. A resonator with a variable coupling would provide a significant improvement: If used to measure a qubit, the coupling to the measurement apparatus could be turned off except during resonator readout, when the coupling could be made large. When coupling two qubits through a resonator, the coupling could be turned on and off as needed $[12,13]$, yielding higher fidelity gates [14,15].

Here we employ an externally controlled variable inductance [16] to modulate the coupling of a resonator to a transmission line, creating the microwave equivalent of a Fabry-Perot cavity with a variable-transparency mirror. The resonator also has fixed coupling to a superconducting phase qubit. We demonstrate the time-controlled release of single-photon Fock and superposition states, thus generating a "flying qubit" [17-19]. We also perform timed capture and release of few-photon coherent states, and use the variable coupling to transmit and release photons with arbitrary waveforms [20,21]. This new capability promises numerous applications in high-fidelity quantum computing and communication.

The schematic in Fig. 1(a) displays a Fabry-Perot cavity, which represents the resonator, with a tunable transparency mirror to represent the variable coupler. A two-level atom plays the role of the qubit. In the actual experiment [Figs. 1(b) and 1(c)], the resonator $(r)$ is a quarterwavelength $(\lambda / 4)$ coplanar waveguide resonator, with one end coupled to a superconducting phase qubit $(q)$ and the other end shorted to ground. Close to the grounded end (a distance $\sim \lambda / 60$ away), the resonator is connected to a variable coupler $(c)$, a transformer comprising two fixed inductors $L_{1}, L_{2}$, and a superconducting quantum interference device (SQUID) with tunable inductance $L_{s}$. A bias (a)

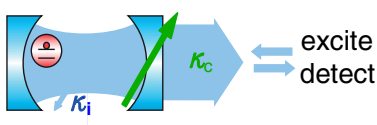

(b)

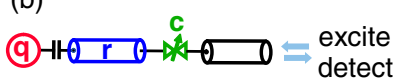

(c)

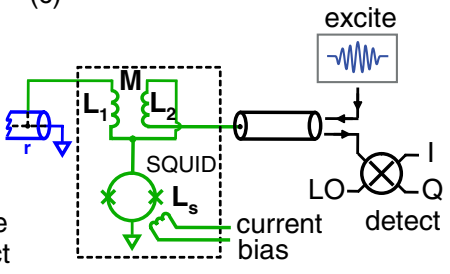

FIG. 1 (color online). Experimental setup. (a) Schematic for a cavity with a variable transparency mirror, where $\kappa_{c}$ is the decay rate through the variable mirror and $\kappa_{i}$ the intrinsic photon decay rate. (b) Experimental schematic. The resonator $r$ is capacitively coupled to a superconducting phase qubit $q$ at the left end, and connected to a transmission line through a variable coupler $c$ at the right end. (c) Details of the variable coupler and the electronic control. Microwave excitation signals drive the resonator through the transmission line, and signals from the resonator are amplified and demodulated using a mixer driven by a local oscillator (LO). The demodulated $I$ and $Q$ signals oscillate at the LO sideband frequency. 
current controls the variable coupler and modulates the coupling to a microwave transmission line by flux tuning the SQUID inductance embedded in the mutual inductance circuit. We want to emphasize that, although a junctiontype variable coupler has been used to couple two qubits [16], here the coupling to the transmission line in place of a qubit requires a complete redesign of the coupling element, as detailed in the Supplemental Material [22].

The resonator-transmission line coupling $\kappa_{c}$ can vary from zero to a maximum emission rate $\kappa_{\max } \approx 1 /(5 \mathrm{~ns})$, over a time scale of a few nanoseconds. The resonator frequency is $f_{r} \simeq 6.57 \mathrm{GHz}$, and the phase qubit has a ground to excited state $(|g\rangle \leftrightarrow|e\rangle)$ transition frequency tunable from $\sim 6$ to $7 \mathrm{GHz}[2,16,23]$. The qubit-resonator coupling, $g / 2 \pi \simeq 12 \mathrm{MHz}$, is calibrated by swap spectroscopy [4], in which the population of qubit excitation is measured as a function of qubit-resonator detuning and interaction time. The qubit-resonator interaction is controlled by tuning the qubit frequency, and is effectively turned off by setting the qubit frequency to its idle point, $400 \mathrm{MHz}$ below the resonator frequency [2,16,23].

The resonator and variable coupler were characterized by measuring the decay of a one-photon Fock state stored in the resonator. When the resonator is weakly coupled to the transmission line, the photon decays due to internal resonator losses, while when strongly coupled, the photon is emitted into the transmission line. The pulse sequence is shown in Fig. 2(a) (top), where the qubit was excited from $|g\rangle$ to $|e\rangle$, and the excitation then swapped to the resonator by an ISWAP pulse, creating a one-photon Fock state [2]. The coupler bias current was then adjusted [Fig. 2(a), middle] from zero to a variable amplitude which set the coupling strength. After a delay time $\tau$, the residual excitation was swapped back to the qubit, and the qubit measured.

Figure 2(b) displays the probability $P_{e}$ of measuring the qubit in $|e\rangle$ as a function of delay $\tau$ and the variable coupler current bias. $P_{e}$ decays exponentially with time $\tau$, with the decay rate varying strongly with coupler bias. Two line cuts are shown in Fig. 2(d), with exponential fits yielding the resonator lifetime $T_{1}$. For zero coupling, as determined by maximizing $T_{1}$ with respect to coupler bias, we find the intrinsic $T_{1, i} \approx 4.5 \mu \mathrm{s}$, in agreement with resonator loss measurements, while for coupling $\kappa_{\text {large }}$ the lifetime was reduced to $T_{1} \approx 30 \mathrm{~ns}$. The resonator inverse lifetime $1 / T_{1}$ is the sum of the intrinsic decay rate $1 / T_{1, i}$ and the coupler emission rate $\kappa_{c}$, so $\kappa_{c}=1 / T_{1}-1 / T_{1, i}$. The coupling dependence on current bias in Fig. 2(b) is in good agreement with calculations, as shown in Fig. 2(c) (see details in the Supplemental Material [22]).

We demonstrated dynamic control by changing the coupling during the delay period, as shown in Fig. 2(e). We started with the coupling set to zero, and after a delay $\tau_{s}$ switched the coupling to $\kappa_{\text {large }} \simeq 1 /(30$ ns) [Fig. 2(a), bottom]. The reduction in the photon lifetime after the switch
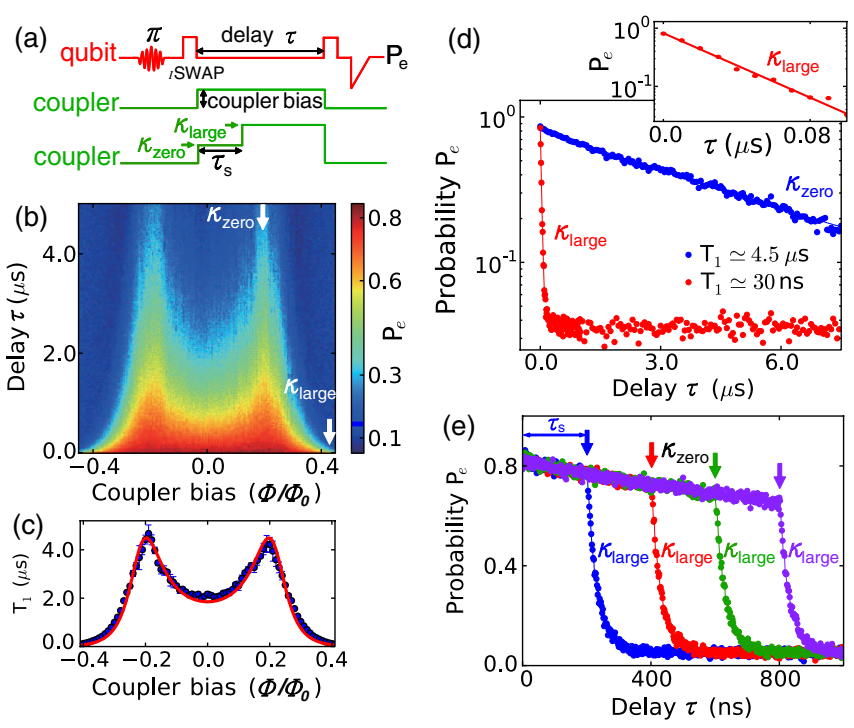

FIG. 2 (color online). Characterization of the variable coupler with Fock state $|1\rangle$. (a) Pulse sequence for the qubit (top) and coupler [middle used in (b) and (c); bottom used in (d)], with details described in the text. The qubit measurement is a projective single-shot procedure, with 600 averages yielding the qubit excited state probability $P_{e}$. (b) $P_{e}$ (color scale) versus $\tau$ (vertical axis) and coupler bias in flux units (horizontal axis). (c) The resonator lifetime $T_{1}$ extracted from data in (b) is displayed as blue dots, compared with the theoretical evaluation of $T_{1}$ (shown as a red line, and see details in the Supplemental Material [22]). (d) Vertical line cuts of (b) display exponential decay of $P_{e}$, which gives resonator lifetime $T_{1}$. Inset shows $P_{e}$ for short times for $\kappa_{\text {large }}$ (e) $P_{e}$ versus delay $\tau$ for coupling strength switching from zero to $\kappa_{\text {large }}$. Decay rate switches from intrinsic lifetime $(4.5 \mu \mathrm{s})$ to $30 \mathrm{~ns}$, with transition taking $\sim 2 \mathrm{~ns}$. Blue, red, green, and purple lines correspond to switching delays $\tau_{s}$ of $200,400,600$, and $800 \mathrm{~ns}$, respectively.

is clearly visible. The upper bound of $\kappa_{\text {zero }}$ is smaller than $0.1 \mathrm{MHz}$ in our system [16]. The coupler switching speed was limited by the $\sim 2 \mathrm{~ns}$ rise time of the coupler bias, roughly 2000 times shorter than $T_{1, i}$.

This measurement does not distinguish between incoherent decay and the expected phase-coherent release of the photon. We therefore also used heterodyne detection, with the resonator "catching" and then "releasing" photons in coherent states. Figure 3(a) displays the pulse sequence: With the coupler set to an intermediate coupling $\kappa_{c}=1 /(356 \mathrm{~ns})$, we excited the resonator with a $100 \mathrm{~ns}$ Gaussian pulse from the microwave source, with the pulse calibrated to trap $\langle n\rangle=10$ photons (see Supplemental Material [22]). The coupling was then set to zero, trapping the photons for a storage time $\tau_{s}$, then set back to $\kappa_{c}=$ $1 /(356 \mathrm{~ns})$, releasing the photons for heterodyne detection.

Figure 3(b) shows the heterodyne-detected signal in the $I$ (real) and $Q$ (imaginary) quadratures in the time domain. During the Gaussian excitation pulse, the signal comprised the reflected component of the excitation. No signal was detected during the subsequent $\tau_{s}=200 \mathrm{~ns}$ storage time 


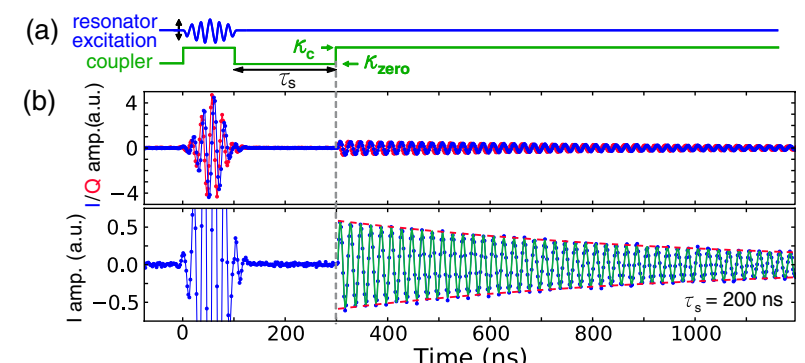

(c)

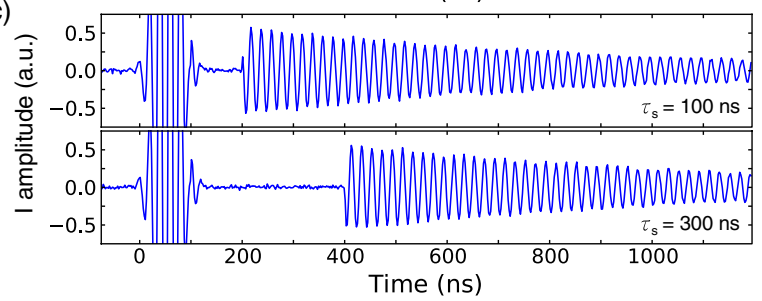

FIG. 3 (color online). Catch and release of photons in coherent states. All traces averaged $10^{5}$ times. (a) Pulse sequence. Resonator (blue) is driven by on-resonance, 100 ns Gaussian excitation pulse, and coupler (green) tuned to $\kappa_{c} \simeq 1 /(356 \mathrm{~ns})$. Photons are stored for time $\tau_{s}$ at zero coupling, then released with $\kappa_{c}=1 /(356 \mathrm{~ns})$. (b) Top panel: Demodulated $I$ and $Q$ quadrature signals for $\tau_{s}=200 \mathrm{~ns}$, with $50 \mathrm{MHz}$ sideband oscillations and a relative $\pi / 2$ phase shift (lines are guides to the eye). Signals include reflected part of excitation pulse, followed by release signal after delay $\tau_{s}$, comprising a sudden onset with exponential decay. Results are given in arbitrary units (a.u.). Bottom panel: I on expanded scale, with sinusoidal fit (thick green line), comprising sideband oscillations with exponential decay envelope (dashed red line, time constant $T_{d}=$ $706 \mathrm{~ns}$ ). The fixed phase with $10^{5}$ averages indicates phase coherence of photon release. (c) I quadrature for trapping delays $\tau_{s}=100$ and $300 \mathrm{~ns}$, showing excitation pulse and a delayed photon release.

with the coupler turned off. A sharp onset followed by an exponentially decaying envelope appeared when the coupler was turned back on, releasing the photons. The signal envelope has a decay time $T_{d}=706 \mathrm{~ns}$, in close agreement with the expected value $2 / \kappa_{c}=712 \mathrm{~ns}$. The amplitude oscillations are from a $50 \mathrm{MHz}$ mixer sideband signal, and the $I$ and $Q$ quadratures have a relative $\pi / 2$ offset, as expected. As the output traces were averaged $10^{5}$ times, the presence of oscillations indicates that the output represents coherent photon release, with a fixed output phase relative to the input. The catch efficiency in this procedure is $13 \%$ (its theoretical estimation is 19\%) and will be optimized in the future [24].

Figure 3(c) displays the $I$ quadratures using storage times $\tau_{s}=100$ and $300 \mathrm{~ns}$. These are identical during the state-generating Gaussian pulse, but during the release the oscillation phase depends on the storage time $\tau_{s}$, scaling as $\sim 0.81 \pi\left(\tau_{s} / 100 \mathrm{~ns}\right)$. This phase accumulation is as expected from the small tuning of the resonator frequency $f_{r}$ with coupler bias (see Supplemental Material [22]), further demonstrating the coherence of the release.
We also calculated the radiated energy $\int_{t_{r}}^{t_{c}}\left[I^{2}(t)+\right.$ $\left.Q^{2}(t)\right] d t$, integrating the signal power from the photon release time $t_{r}$ to a cutoff $t_{c}=t_{r}+3 T_{d}$. We find that the released energy for $\tau_{s}=300 \mathrm{~ns}$ is $4 \%$ lower than for $\tau_{s}=100 \mathrm{~ns}$, in agreement with the expected intrinsic resonator loss.

These measurements confirm the phase-coherent capture and release of coherent states. To demonstrate that we can achieve the same control for nonclassical states, we used the qubit to generate [2] the photon superposition state $(|0\rangle+|1\rangle) / \sqrt{2}$ and measured the release signal after turning on the coupling [Fig. 4(a)]. For an intermediate coupling $\kappa_{c} \simeq 1 /(320 \mathrm{~ns})$ and a large coupling $\kappa_{c} \simeq$ $1 /(30 \mathrm{~ns})$, the signal's exponential decay envelope has a time constant $T_{d} \approx 625$ and $69 \mathrm{~ns}$, respectively, close to the expected $2 / \kappa_{c}$, verifying that the coupling determines
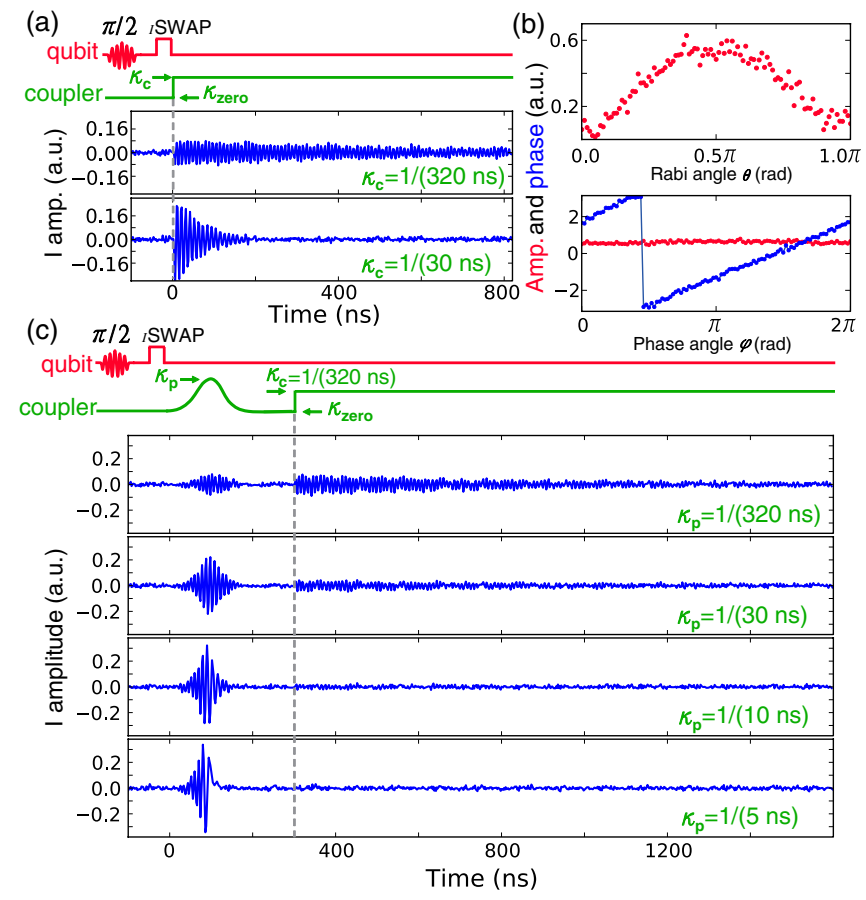

FIG. 4 (color online). Storage, release, and heterodyne detection of nonclassical photon states. (a) Top: Pulse sequence to prepare a superposition Fock state $(|0\rangle+|1\rangle) / \sqrt{2}$ and release this nonclassical photon state. Coupler is switched at $t=0$ from zero to a coupling $\kappa_{c}$. Middle and bottom: Heterodyne-detected $I$ quadrature for two $\kappa_{c}$ values. Mixer LO sideband frequency is $110 \mathrm{MHz}$, with $10^{6}$ averages. (b) Amplitude (red) and phase (blue) of demodulated signal for the released superposition state $\cos (\theta / 2)|0\rangle+e^{i \phi} \sin (\theta / 2)|1\rangle$. The measurement is taken with $3.6 \times 10^{5}$ averages. (c) First panel: Tailored, two-segment release pulse sequence for $(|0\rangle+|1\rangle) / \sqrt{2}$ superposition state, using a 200 ns Gaussian control pulse followed by a rectangular pulse, with intervening 100 ns delay. Second to fifth panels: Pulse amplitude $\kappa_{p}$ set to different values as shown. I quadrature amplitude depends on $\kappa_{p}$, with different amounts of energy released during Gaussian pulse; two top panels show remainder released during final rectangular pulse. 
the release rate. The integrated energy for intermediate coupling is 7\% lower than for large coupling, attributed to greater intrinsic loss from the slower release.

We next tested the release and detection of the qubitprepared superposition state $\cos (\theta / 2)|0\rangle+e^{i \phi} \sin (\theta / 2)|1\rangle$, akin to previous work with static coupling $[17,18]$. After state preparation, the variable coupling was changed from zero to $\kappa_{c}=1 /(320 \mathrm{~ns})$, and the released photons were heterodyne detected as a function of the Rabi angle $\theta$ and the phase angle $\phi$, with Fourier transforms of $I$ and $Q$ yielding the signal amplitude and phase. Figure 4(b) shows the dependence of the signal amplitude on $\theta$, with $\phi=0$. The maximum amplitude is at $\theta=\pi / 2$, corresponding to $(|0\rangle+|1\rangle) / \sqrt{2}$. The amplitude goes to zero for the pure Fock states at $\theta=0$ and $\pi$ as expected, due to the loss of phase coherence at the Bloch sphere poles. When releasing the state $\left(|0\rangle+e^{i \phi}|1\rangle\right) / \sqrt{2}$ with $\theta=\pi / 2$ and varying $\phi$, the signal has constant amplitude $(0.613 \pm 0.066)$ and phase increasing linearly with $\phi$.

The on-demand, real-time gating of the coupler enables precise shaping of the photon release waveform. Figure 4(c) shows the tailored time-dependent release of the $(|0\rangle+$ $|1\rangle) / \sqrt{2}$ photon state, modulating the coupling with a $200 \mathrm{~ns}$ Gaussian bias pulse with peak coupling $\kappa_{p}$, followed by a $100 \mathrm{~ns}$ delay and then completing the release with $\kappa_{c}=1 /(320 \mathrm{~ns})$. Figure 4(c) shows the $I$ quadrature signal for $\kappa_{p}=1 /(320 \mathrm{~ns}), 1 /(30 \mathrm{~ns}), 1 /(10 \mathrm{~ns})$, and $1 /(5 \mathrm{~ns})$, with a Gaussian-like release waveform mimicking the coupler pulse. For the top three panels, energy integrals show that 17.5, 43.1, and $100 \%$ of the total stored energy is released during the pulse, with the remainder released after the $100 \mathrm{~ns}$ delay. For couplings $\kappa_{p} \gtrsim$ $1 /(10 \mathrm{~ns})$, the release is completed during the Gaussian pulse. In contrast to fixed coupling, in which the waveform decays exponentially with time, this experiment shows carefully shaped waveforms, a critical feature needed for high-fidelity transfer of photonic information [14,15].

In conclusion, we have realized a superconducting resonator with a completely controllable mirror transparency. While an adjustable cavity-environment coupling has been speculated using interference effect $[25,26]$ or manipulation of energy levels [27], this is the first experimental demonstration of a variable transparency mirror in any system. We have demonstrated the phase-coherent, controlled capture and release of coherent and superposition photon states from a resonator, using a resonatortransmission line variable coupling. The catch and release of photons has attracted significant attention in atomic and planar photonic systems [20,26,27]. Our work extends prior coherent state demonstrations to the microwave domain, along with release of nonclassical photon states with a more complete characterization. This powerful technique should allow long-range entanglement [4,28-32], where the shaped release we display in the last experiment is a key ingredient for high-fidelity state transfer $[14,15]$.
This capability will further enable tunable coupling for resonator-based dispersive qubit readout, where timedomain control can minimize deleterious dephasing while maximizing measurement bandwidth and signal strength.

This work was supported by IARPA under ARO Grant No. W911NF-08-01-0336 and under ARO Grant No. W911NF-09-1-0375. M.M. acknowledges support from an Elings Postdoctoral Fellowship. R. B. acknowledges support from the Rubicon program of the Netherlands Organization for Scientific Research. Devices were made at the UC Santa Barbara Nanofabrication Facility, a part of the NSF funded National Nanotechnology Infrastructure Network.

*Present address: Department of Physics, Zhejiang University, Hangzhou 310027, China.

martinis@physics.ucsb.edu

[1] M. Hofheinz, E. M. Weig, M. Ansmann, R. C. Bialczak, E. Lucero, M. Neeley, A. D. O'Connell, H. Wang, J. M. Martinis, and A. N. Cleland, Nature (London) 454, 310 (2008).

[2] M. Hofheinz et al., Nature (London) 459, 546 (2009).

[3] H. Wang et al., Phys. Rev. Lett. 103, 200404 (2009).

[4] M. Mariantoni et al., Nat. Phys. 7, 287 (2011).

[5] A. Blais, R.-S. Huang, A. Wallraff, S. M. Girvin, and R. J. Schoelkopf, Phys. Rev. A 69, 062320 (2004).

[6] A. Wallraff, D. Schuster, A. Blais, L. Frunzio, J. Majer, M. Devoret, S. Girvin, and R. Schoelkopf, Phys. Rev. Lett. 95, 060501 (2005).

[7] Y. Kubo et al., Phys. Rev. Lett. 105, 140502 (2010).

[8] D. I. Schuster et al., Phys. Rev. Lett. 105, 140501 (2010).

[9] F. Mallet, F. R. Ong, A. Palacios-Laloy, F. Nguyen, P. Bertet, D. Vion, and D. Esteve, Nat. Phys. 5, 791 (2009).

[10] B. R. Johnson, et al., Nat. Phys. 6, 663 (2010).

[11] P. J. Leek, M. Baur, J. M. Fink, R. Bianchetti, L. Steffen, S. Filipp, and A. Wallraff, Phys. Rev. Lett. 104, 100504 (2010).

[12] M. S. Allman, F. Altomare, J. D. Whittaker, K. Cicak, D. Li, A. Sirois, J. Strong, J. D. Teufel, and R. W. Simmonds, Phys. Rev. Lett. 104, 177004 (2010).

[13] S. J. Srinivasan, A. J. Hoffman, J. M. Gambetta, and A. A. Houck, Phys. Rev. Lett. 106, 083601 (2011).

[14] J. I. Cirac, P. Zoller, H. J. Kimble, and H. Mabuchi, Phys. Rev. Lett. 78, 3221 (1997).

[15] A. N. Korotkov, Phys. Rev. B 84, 014510 (2011).

[16] R. C. Bialczak et al., Phys. Rev. Lett. 106, 060501 (2011).

[17] D. Bozyigit et al., Nat. Phys. 7, 154 (2011).

[18] A. A. Houck et al., Nature (London) 449, 328 (2007).

[19] D. P. Divincenzo, Fortschr. Phys. 48, 771 (2000).

[20] M. D. Lukin, Rev. Mod. Phys. 75, 457 (2003).

[21] M. Keller, B. Lange, K. Hayasaka, W. Lange, and H. Walther, Nature (London) 431, 1075 (2004).

[22] See Supplemental Material at http://link.aps.org/ supplemental/10.1103/PhysRevLett.110.107001 for details of the coupler design, theoretical modeling of the coupler, and calibration of trapped photons.

[23] Yi Yin et al., Phys. Rev. A 85, 023826 (2012). 
[24] A theoretical model of the catch efficiency is developed by J. Wenner et al. (unpublished).

[25] Y. Tanaka, J. Upham, T. Nagashima, T. Sugiya, T. Asano, and S. Noda, Nat. Mater. 6, 862 (2007).

[26] Q. Xu, P. Dong, and M. Lipson, Nat. Phys. 3, 406 (2007).

[27] T. Tanabe, M. Notomi, H. Taniyama, and E. Kuramochi, Phys. Rev. Lett. 102, 043907 (2009).

[28] H. Wang et al., Phys. Rev. Lett. 106, 060401 (2011).
[29] M. Neeley et al., Nature (London) 467, 570 (2010).

[30] S. Ritter, C. Nölleke, C. Hahn, A. Reiserer, A. Neuzner, M. Uphoff, M. Mücke, E. Figueroa, J. Bochmann, and G. Rempe, Nature (London) 484, 195 (2012).

[31] L.-M. Duan, M. D. Lukin, J. I. Cirac, and P. Zoller, Nature (London) 414, 413 (2001).

[32] H.-J. Briegel, W. Dür, J. I. Cirac, and P. Zoller, Phys. Rev. Lett. 81, 5932 (1998). 